\title{
Resistance of Fetal Guinea Pig Ventricular Myocardium to Hypoxia: Maintained Intracellular ATP Prevents the Opening of ATP-Sensitive Potassium Channels
}

\author{
Naoki Agata, Yoshimitsu Kato, Shogo Hamaguchi,* Iyuki Namekata, and Hikaru Tanaka \\ Department of Pharmacology, Faculty of Pharmaceutical Sciences, Toho University; 2-2-1 Miyama, Funabashi, \\ Chiba 274-8510, Japan. \\ Received September 29, 2018; accepted November 12, 2018
}

\begin{abstract}
The presence and function of the ATP-sensitive potassium channel current $\left(I_{\text {KATP }}\right)$ were examined in the guinea pig myocardium to clarify the mechanisms for the resistance of the fetal myocardium to hypoxia. Experimental hypoxia markedly reduced the action potential duration and contractile force in isolated ventricular myocardium from the adult, but only moderately in those from the fetus. In isolated ventricular cardiomyocytes, the density of the $I_{K A T P}$ activated by cromakalim, as well as their sensitivity to intracellular ATP concentration, were not different between the fetus and adult. The tissue ATP content was similar between the fetal and adult myocardium under normal condition, but the hypoxia-induced decrease was smaller in the fetus. Confocal microscopic analysis revealed that the mitochondria in the fetal cardiomyocyte is less in quantity than that in the adult and is more localized to the cell center. These results indicate that $I_{\text {KATP }}$ in the fetal guinea pig myocardium has a current density and ATP sensitivity similar to those of the adult, but is not activated under hypoxic conditions because the energy metabolism of the fetal myocardium is less dependent on oxidative phosphorylation.
\end{abstract}

Key words fetus; guinea pig; ventricle; ATP-sensitive $\mathrm{K}^{+}$channel; action potential duration

\section{INTRODUCTION}

Changes in the microstructural, biochemical and physiological properties of the myocardium take place during development of the heart. ${ }^{1-3)}$ Changes in excitation-contraction mechanisms of the myocardium has been reported in many experimental animal species such as the rabbit, rat and mouse. The guinea pig myocardium, which has an action potential with a plateau phase resembling that of the human myocardium, has been extensively used in cardiac research. However, information on the developmental changes in the guinea pig myocardium is limited. We have shown that the excitation-contraction mechanism of the guinea pig ventricular myocardium changes during late embryonic development from sarcolemma-dependent $\mathrm{Ca}^{2+}$ handling to sarcoplasmic reticulum-dependent $\mathrm{Ca}^{2+}$ handling. $\left.{ }^{4}\right)$

Concerning the action potential, we have shown that the action potential duration moderately decreases during the late prenatal period and recovers during postnatal development. ${ }^{5)}$ This was accompanied by developmental increases in the Ltype calcium current and the delayed rectifier potassium current $\left.\left(I_{\mathrm{Kr}}\right) \cdot{ }^{6}\right)$ The change in action potential duration could be explained by the difference in the developmental time course of increase between the two currents. ${ }^{7}$

The ATP-sensitive potassium channel is present in cardiomyocytes and is activated under hypoxic conditions and by pharmacological agents. ${ }^{8-12}$ The ATP-sensitive potassium channel current $\left(I_{\text {КАTP }}\right)$ has a linear voltage dependence and thus generates a potent repolarizing power at depolarized membrane potentials. $I_{\text {KATP }}$ is responsible for the hypoxiainduced shortening of the myocardial action potential and decrease in contraction. We found that the contractile force and action potential duration of the fetal guinea-pig ventricular myocardium is relatively resistant to experimental hypoxia. ${ }^{13)}$ Although this could be the result of a reduced density of $I_{\text {KATP }}$ in the fetal cardiomyocyte and/or of the characteristic fetal energy metabolism, the presence and regulation of $I_{\text {KATP }}$ in the fetal guinea pig myocardium has not yet been clarified. In the present study, we examined the basic properties of the $I_{\text {KATP }}$ and the energy metabolism in the fetal guinea pig myocardium to clarify the mechanisms for its resistance to hypoxia.

\section{MATERIALS AND METHODS}

Animals The present study was conducted in accordance with the Guiding "Principles for the Care and Use of Laboratory Animals Approved by The Japanese Pharmacological Society" and the Guide for the Care and Use of Laboratory Animals at Faculty of Pharmaceutical Sciences, Toho University. Fetal (44 to $55 \mathrm{~d}$ after conception) and adult (45 to $60 \mathrm{~d}$ after birth) guinea pigs were used. The animals were anesthetized with pentobarbital and the hearts were removed after cervical dislocation. The fetuses were delivered by Caesarean section.

Measurement of Action Potential and Contractile Force Standard glass microelectrode experiments and contractile force measurements were performed with isolated right ventricular strips as described. ${ }^{13)}$ The preparations were placed in an organ bath containing physiological salt solution maintained at $37^{\circ} \mathrm{C}(\mathrm{pH} 7.4)$ and driven at $1 \mathrm{~Hz}$ by field electrical stimulation. The composition of the solution was as follows $(\mathrm{mM}): \mathrm{NaCl} 118.4, \mathrm{KCl} 4.7, \mathrm{CaCl}_{2} 2.5, \mathrm{MgCl}_{2} 1.2, \mathrm{KH}_{2} \mathrm{PO}_{4}$ 1.2, $\mathrm{NaHCO}_{3}$ 24.9, and glucose 11.1. For the measurements under normal and hypoxic condition, the solution was gassed with $95 \% \mathrm{O}_{2}-5 \% \mathrm{CO}_{2}$ and $95 \% \mathrm{~N}_{2}-5 \% \mathrm{CO}_{2}$, respectively. Action potentials were recorded from the endocardial surface with glass microelectrodes filled with $3 \mathrm{M} \mathrm{KCl}$ (resistance 
15 to $50 \mathrm{M} \Omega$ ) and connected to a high impedance amplifier (MEZ7101, Nihon Kohden, Tokyo, Japan). For measurement of contractile force, the preparation was attached to a forcedisplacement transducer (TB-611T, Nihon Kohden) connected to a carrier amplifier (AP-621G, Nihon Kohden). The output of the amplifiers was digitized and analyzed (Power Lab System, AD Instruments, Dunedin, New Zealand). Concerning the action potentials, the time for $50 \%$ repolarization was measured.

Measurement of Potassium Current Ventricular cardiomyocytes from the fetus and adult were obtained by Langendorff perfusion and collagenase digestion of the myocardial tissue as described. ${ }^{6)}$ For potassium current measurements, the cells were superfused with Tyrode's solution of the following composition (mM): $\mathrm{NaCl} 143, \mathrm{KCl} 5.4, \mathrm{MgCl}_{2}$ 1.0, $\mathrm{CaCl}_{2}$ 1.8, $\mathrm{NaH}_{2} \mathrm{PO}_{4} 0.33$, glucose 5.5, and $N$-(2-hydroxyethyl)piperazine$N^{\prime}$-2-ethanesulfonic acid (HEPES) 5.0 (pH 7.4). Nisoldipine $(1 \mu \mathrm{M})$ was added to block the $\mathrm{Ca}^{2+}$ current. The solution was gassed with $100 \% \mathrm{O}_{2}$ and warmed to $37^{\circ} \mathrm{C}$. The glass micropipettes contained the following solution $(\mathrm{mM})$ : $\mathrm{KCl} 130$, HEPES $5, \mathrm{MgCl}_{2} 1, \mathrm{~K}_{2}$ ATP 2 or 5, ethylene glycol bis(2-aminoethyl ether)- $N, N, N^{\prime}, N^{\prime}$-tetraacetic acid (EGTA) 1 (titrated to $\mathrm{pH} 7.2$ with $\mathrm{KOH}$ ) and had a resistance of 3 to $5 \mathrm{M} \Omega$.

Measurement of ATP Concentration Tissue ATP concentration was measured as described earlier. ${ }^{11,14)}$ The myocardial tissue preparations in the organ bath were freeze clamped with an aluminum tongue chilled with liquid $\mathrm{N}_{2}$. The frozen muscles were pulverized and homogenized. The ATP content was determined with the firefly luminescence method. ${ }^{12)}$
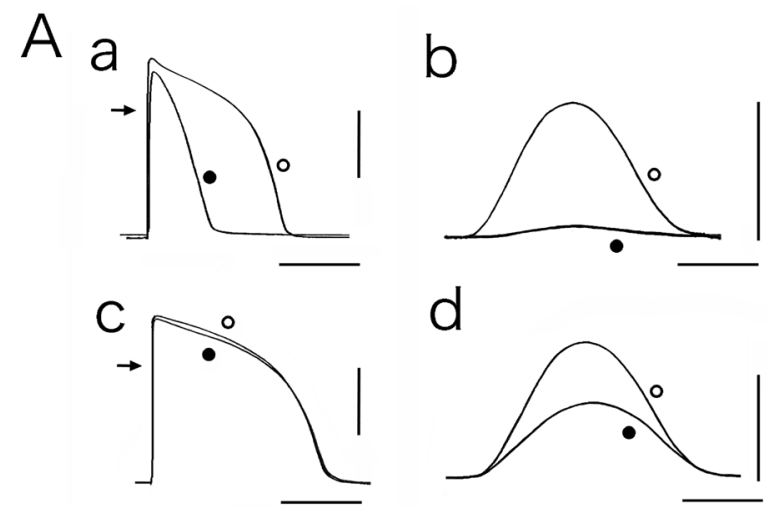

$\mathrm{B}$

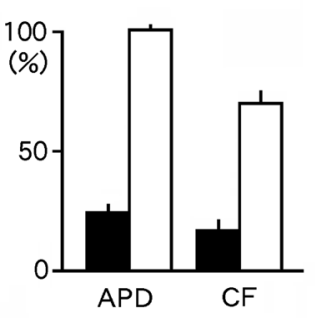

Fig. 1. Effect of Hypoxia on the Electrical and Contractile Function of Adult and Fetal Ventricular Muscles

A: Typical recordings of the action potential $(a, c)$ and contractile force $(b, d)$ of adult $(a, b)$ and fetal $(c, d)$ ventricular myocardium under normal condition (open circles) and after $60 \mathrm{~min}$ exposure to hypoxic condition (closed circles). Vertical and horizontal bars in a and c indicate $50 \mathrm{mV}$ and $100 \mathrm{~ms}$, respectively, and arrows indicate $0 \mathrm{mV}$. Vertical and horizontal bars in b and d indicate $100 \mathrm{mg}$ and $100 \mathrm{~ms}$, respectively. B: Summarized results for the action potential duration at $50 \%$ repolarization (APD) and contractile force (CF) under hypoxic condition of the adult (closed columns) and fetal (open columns) myocardia. Values under hypoxic condition were expressed as percentages of the value under normal condition. Columns and vertical bars in B indicate the mean \pm S.E.M. from 5 experiments.
Confocal Imaging Analysis The morphology of the sarcolemma and the intracellular localization of mitochondria were determined in cardiomyocytes by confocal microscopy. Isolated cardiomyocytes were loaded simultaneously with the membrane dye PKH67 and the mitochondria dye tetramethyl rhodamine ethylester (TMRE). The cells were placed in a glass bottom chamber loaded on a confocal microscope (A1R; Nikon, Tokyo, Japan) and the membrane and mitochondria were observed under excitation at $488 \mathrm{~nm}$ and $514 \mathrm{~nm}$, respectively. Confocal images were obtained in $512 \times 512$ pixels and the fraction of PKH67 positive and TMRE positive pixels in a cell was calculated as described. ${ }^{15)}$

Statistics and Chemicals All experimental data are expressed as the mean \pm standard error of the mean (S.E.M.) and statistical significance of differences between means were evaluated by the unpaired or paired $t$-test. A $p$ value less than 0.05 were considered significant. The agents used were cromakalim (Sigma-Aldrich, St. Louis, MO, U.S.A.), dinitrophenol (Sigma-Aldrich), glibenclamide (Sigma-Aldrich), TMRE (Thermo Fisher Scientific, Waltham, MA, U.S.A.) and PKH67 Fluorescent Cell Linker Kits (Sigma-Aldrich). All other agents and chemicals were commercial products of the highest available quality.

\section{RESULTS}

Effect of Hypoxia on the Action Potential and Contractile Force The action potentials of the adult and fetal ven-
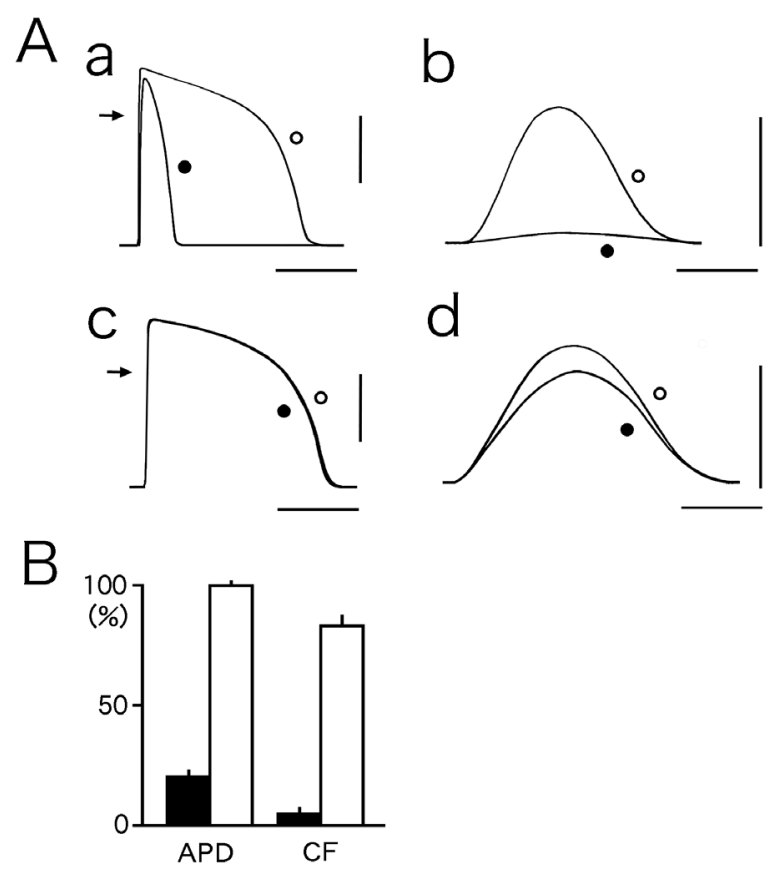

Fig. 2. Effect of Dinitrophenol on the Electrical and Contractile Function of Adult and Fetal Ventricular Muscles

A: Typical recordings of the action potential $(a, c)$ and contractile force $(b, d)$ of adult (a, b) and fetal (c, d) ventricular myocardium before (open circles) and at $60 \mathrm{~min}$ after the application of $10 \mu \mathrm{M}$ dinitrophenol (closed circles). Vertical and horizontal bars in a and c indicate $50 \mathrm{mV}$ and $100 \mathrm{~ms}$, respectively, and arrows indicate $0 \mathrm{mV}$. Vertical and horizontal bars in b and d indicate $100 \mathrm{mg}$ and $100 \mathrm{~ms}$, respectively. B: Summarized results for the action potential duration at $50 \%$ repolarization (APD) and contractile force (CF) before (open circles) and at $60 \mathrm{~min}$ after the application of $10 \mu \mathrm{M}$ dinitrophenol (closed circles) of the adult (closed columns) and fetal (open columns) myocardia. Values in the presence of dinitrophenol were expressed as percentages of the values in its absence. Columns and vertical bars in $\mathrm{B}$ indicate the mean \pm S.E.M. from 5 experiments. 
tricular myocardium and the effects of hypoxia were obtained by glass microelectrodes (Fig. 1). The action potential duration of the fetal myocardium was slightly longer than that of the adult (Figs. 1Aa, Ac); action potential duration at 50\% repolarization in the adult and fetal ventricular myocardium was $175.2 \pm 7.6(n=10)$ and $187.4 \pm 10.4 \mathrm{~ms}(n=10)$, respectively. In the adult myocardium, hypoxia markedly decreased the action potential duration as well as contractile force (Figs. 1Aa, $\mathrm{Ab}, 1 \mathrm{~B})$. In the fetal myocardium, action potential duration was completely preserved under hypoxia and about $70 \%$ of the contractile force was maintained (Figs. 1Ac, Ad, 1B).

Effect of Dinitrophenol on the Action Potential and Contractile Force In the adult myocardium, dinitrophenol, an uncoupler of oxidative phosphorylation, markedly decreased the action potential duration as well as contractile force (Figs. $2 \mathrm{Aa}, \mathrm{Ab}, 2 \mathrm{~B})$. In the fetal myocardium, action potential duration was completely preserved under dinitrophenol and about $70 \%$ of the contractile force was maintained. The action potential duration and contractile force under dinitrophenol were significantly larger in the fetus than in the adult (Figs. 2Ac, Ad, 2B).

Measurement of $I_{\mathrm{KATP}}$ and the Effect of Intracellular ATP Concentration $I_{\mathrm{KATP}}$ was measured in isolated ventricular cardiomyocytes from the adult and fetus (Fig. 3). Both in adult and fetal myocytes, the background current showed inward rectification; the current reversed at -85 to $-90 \mathrm{mV}$ and the outward current peaked at $-70 \mathrm{mV}$. $I_{\text {KATP }}$ was induced by $30 \mu \mathrm{M}$ cromakalim, an ATP-sensitive potassium channel opener. The current density was larger with an intracellular ATP concentration of $2 \mathrm{mM}$ (Figs. 3A, 3B) than with $5 \mathrm{mM}$ (Figs. 3C, 3D). The outward current density at the end of a depolarizing pulse to $10 \mathrm{mV}$ in adult and fetal cardiomyocytes were $43.8 \pm 8.0(n=6)$ and $37.5 \pm 4.5 \mathrm{pA} / \mathrm{pF}(n=6)$, respectively, when measured with $2 \mathrm{mM}$ intracellular ATP, and $5.5 \pm 2.9$ $(n=6)$ and $11.2 \pm 2.7 \mathrm{pA} / \mathrm{pF}(n=6)$, respectively, when measured with $5 \mathrm{mM}$ intracellular ATP. $I_{\mathrm{KATP}}$ was completely inhibited by $10 \mu \mathrm{M}$ glybenclamide, an ATP-sensitive potassium channel blocker. There was no significant difference in current density between the adult and fetus under corresponding intracellular ATP concentration.

Effect of Hypoxia on ATP Content The tissue ATP content was measured under the same condition as that for action potential and contractile force measurements (Fig. 4). The ATP content was similar under normal condition between the adult and fetal myocardium. The ATP content was markedly decreased under hypoxia in the adult myocardium. In the fetal myocardium, the decrease was smaller than in the adult. The residual ATP content under hypoxia was significantly larger in
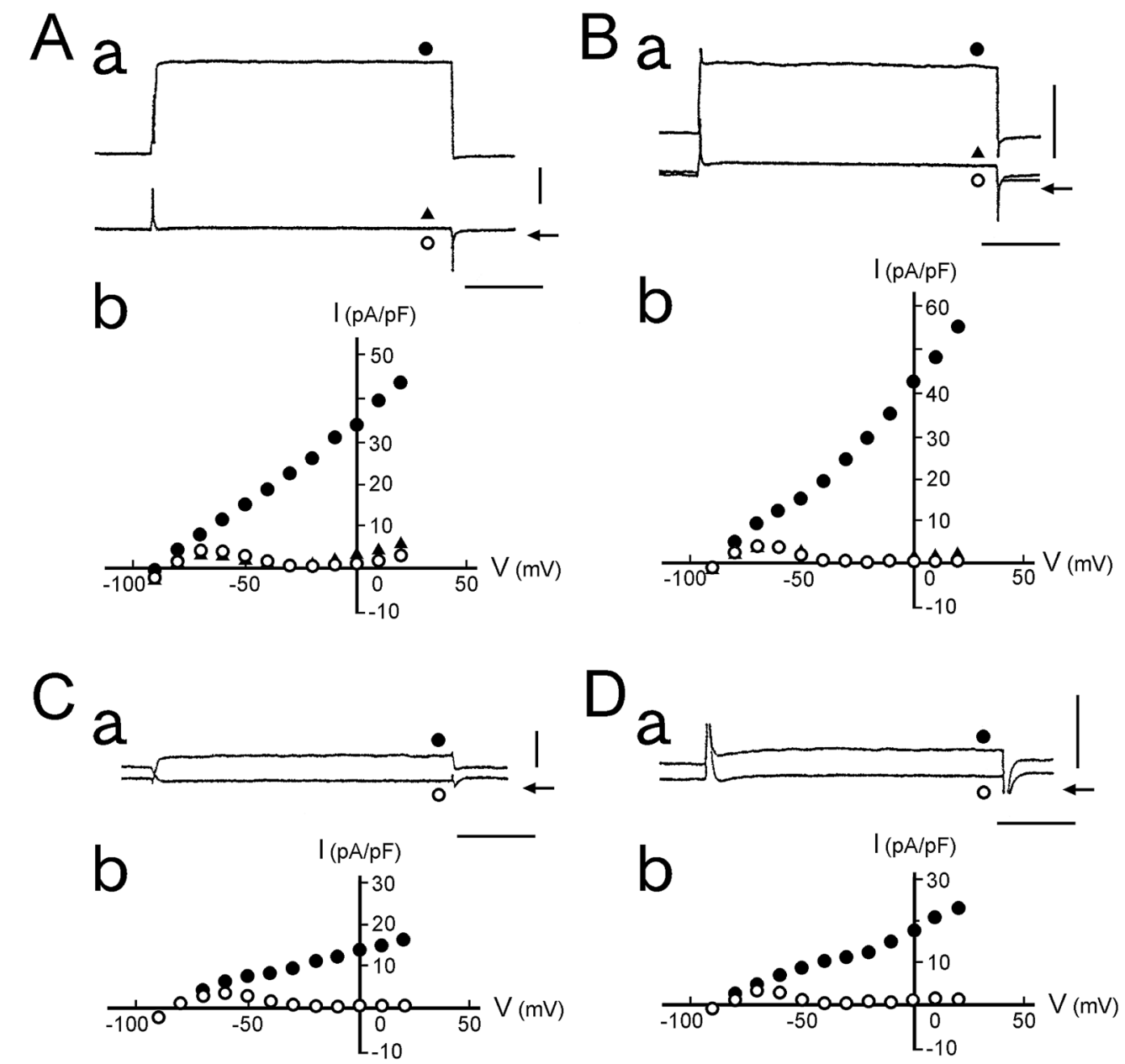

Fig. 3. $I_{\text {KATP }}$ in Adult and Fetal Myocytes

$I_{\text {KATP }}$ was measured in adult (A, C) and fetal (B, D) ventricular cardiomyocytes with $2 \mathrm{mM}$ (A, B) or $5 \mathrm{mM}$ (C, D) intrapippette ATP. Typical current recordings (a) on depolarization to $+10 \mathrm{mV}$ from a holding potential of $-40 \mathrm{mV}$ in the absence of agents (open circles), in the presence of $30 \mu \mathrm{M}$ cromakalim (closed circles), and in the presence of both $30 \mu \mathrm{M}$ cromakalim and $10 \mu \mathrm{M}$ glicanclamide (closed triangles). Vertical and horizontal bars indicate $1 \mathrm{nA}$ and $50 \mathrm{~ms}$, respectively, and arrows indicate 0 current level. Current-voltage relationships (b) of the currents at the end of $200 \mathrm{~ms}$ voltage clamp pulses. 


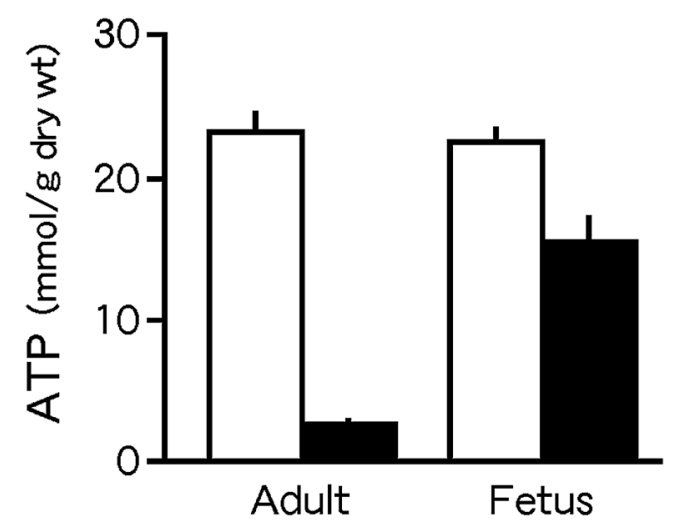

Fig. 4. Tissue ATP Content in Adult and Fetal Myocardium under Normal Condition (Open Columns) and after $60 \mathrm{~min}$ Exposure to Hypoxic (Closed Columns) Condition

Columns and vertical bars indicate the mean \pm S.E.M. from 6 experiments.
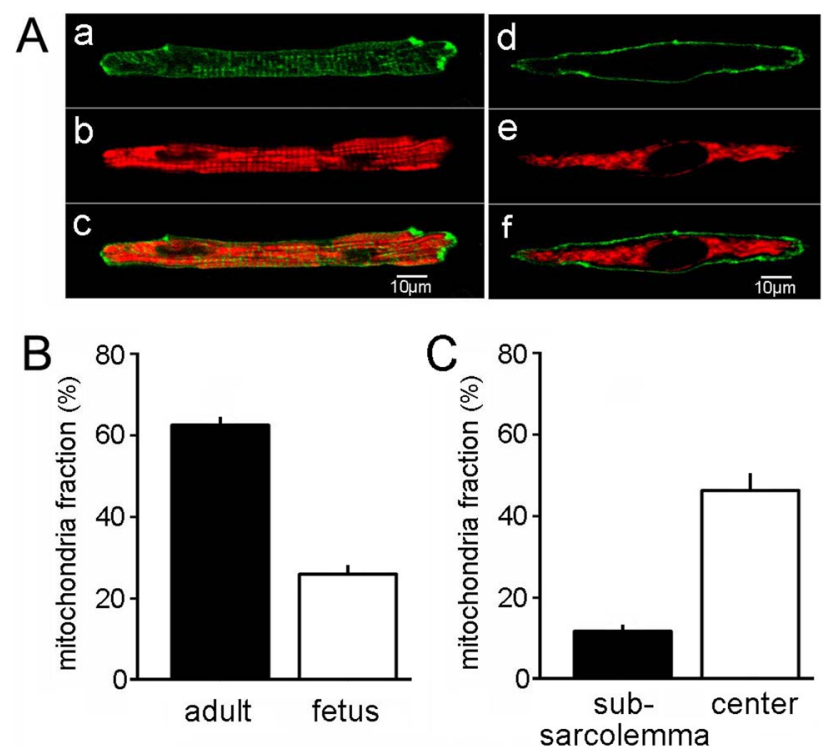

Fig. 5. Microstructure of Adult and Fetal Cardiomyocytes

A: Confocal microscopic images of adult $(a, b, c)$ and fetal $(d, e, f)$ cardiomyocytes stained with the membrane probe PKH67 (a, d), the mitochondria probe TMRE (b, e) and their merge (c, f). B: Mitochondria fraction calculated as the fraction of TMRE fluorescence-positive pixels within the whole cytoplasmic region in each cell. C: Mitochondria fraction of fetal cardiomyocytes calculated as the fraction of TMRE fluorescence-positive pixels in the subsarcolemmal region within $1 \mu \mathrm{m}$ from the sarcolemma (subsarcolemma) and the other regions closer to the center of the cell (center). Columns and vertical bars indicate the mean \pm S.E.M. from 6 cells. (Color figure can be accessed in the online version.)

the fetus than in the adult.

Morphology of the Sarcolemma and Intracellular Localization of Mitochondria The sarcolemma and mitochondria of the cardiomyocytes were observed with confocal microscopy (Fig. 5). In the adult cardiomyocytes, T-tubules, transverse invaginations of the sarcolemma, were present at regular intervals throughout the cell. The mitochondria were present throughout the cytoplasm. Thus, the sarcolemma and the mitochondria were in proximity throughout the cell. In contrast, the fetal cardiomyocytes lacked T-tubules and the mitochondria tended to localize in the cell central region far from the sarcolemma. This means that the sarcolemma and the mitochondria were more separated in the fetal cardiomyocyte than in the adult. The cellular mitochondria content, as evaluated as the fraction of TMRE positive pixels in the total cell area, was significantly lower in the fetal cardiomyocyte than in the adult.

\section{DISCUSSION}

The present study was undertaken to clarify the mechanisms for the resistance of the fetal myocardium to hypoxia. The fetal guinea pig myocardium was relatively resistant to hypoxia when compared to the adult; the action potential duration as well as the contractile force was maintained under hypoxia (Fig. 1). The shortening of the action potential duration following a decline in oxygen support has been attributed to the opening of ATP-sensitive potassium channels. ${ }^{8-12)} \mathrm{We}$ reported that the hypoxia-induced decline in action potential duration of the adult guinea pig ventricular myocardium was partially attenuated by the ATP-sensitive potassium channel blocker, glybenclamide. ${ }^{13)}$ Thus it was possible that the resistance of guinea pig fetal myocardium to hypoxia was due to a lack of the ATP-sensitive potassium channel. Voltage clamp measurement of $I_{\mathrm{KATP}}$, however, revealed that the current density was not different between adult and fetal cardiomyocytes (Fig. 3). This is consistent with our previous observation that cromakalim, an ATP-sensitive potassium channel opener, markedly shortened the action potential duration both in the adult and fetal guinea pig myocardium with a similar efficacy and time course. ${ }^{13)}$ Thus, the resistance of the fetal myocardium could not be ascribed to lack of ATP-sensitive potassium channels.

The sensitivity to intracellular ATP of the fetal ATP-sensitive potassium channel was not different from that of the adult (Fig. 3). On the other hand, the fetal myocardium was resistant not only to hypoxia but also to the metabolic inhibitor, dinitrophenol (Fig. 2). These results suggest that the cellular energy metabolism of the fetal myocardia may be different from that of the adult. Measurement of tissue ATP content showed that the ATP synthesizing system of the fetal myocardium is indeed resistant to hypoxia (Fig. 4). It is generally understood that the ATP synthesis of the developing myocardium is less dependent on mitochondrial oxidative phosphorylation than that of the adult, but is more dependent on glycolysis. ${ }^{16)}$ Concerning the guinea pig, it was reported on the basis of enzyme and metabolite analysis that the fetal and neonatal hearts have a high anaerobic glycolytic capacity, which decreases during postnatal development. ${ }^{17,18)}$ Glycolytically-derived ATP was estimated to be 3 to 4 fold higher in the fetal than the adult heart. ${ }^{18)}$ Histological and microstructural observations in the literature $^{19}$ ) indicate large difference in the composition of the cardiomyocytes. The adult cardiomyocyte is abundant in mitochondria with well-developed cristae, while the developing cardiomyocyte have less mitochondria but is abundant in glycogen particles. Thus, the resistance of fetal guinea pig myocardium to hypoxia could be ascribed to the low dependence of its energy metabolism on oxidative phosphorylation. This agrees with the prediction based on computational analysis that fetal ventricular myocytes with high glycogen content, higher hexokinase activity and lower total creatine concentration shows prolonged maintenance of contraction under anoxic condition. ${ }^{20)}$ Such dependence on glycolysis rather than on oxidative phosphorylation has also been reported in the cultured cardiomyocytes ${ }^{21)}$ and in undifferentiated pluripotent stem cell-derived cardiomyocytes. ${ }^{22)}$ Variation in the energy 
metabolism of cardiomyocytes may affect their sensitivity to hypoxia and to certain pharmacological agents.

Inhibition of oxidative phosphorylation by dinitrophenol in fetal myocardia induced a partial decrease in the contractile force, but did not affect action potential duration at all (Fig. 2). This is consistent with earlier studies which provided functional evidence that glycolysis is more effective than oxidative phosphorylation in preventing the opening of the ATPsensitive potassium channels and conversely, energy provided by oxidative phosphorylation preferentially supports contractile function. ${ }^{23-25)}$ In the fetal guinea pig cardiomyocyte, the mitochondria were localized in the central region of the cell and were absent in the subsarcolemmal region, which should have the largest influence on the activity of membrane channels and transporters (Fig. 5). This contrasts with the situation in the adult cardiomyocyte in which the mitochondria present throughout the cytoplasm including the subsarcolemmal region and the well-developed T-tubules ensure the adjacency of the sarcolemma and the mitochondria. Thus, not only the relative quantity of different cellular components for energy metabolism but also their positional relationship with the sarcolemma may affect the sensitivity of cellular processes to metabolic inhibition by hypoxia and certain pharmacological agents.

In conclusion, $I_{\text {KATP }}$ in the fetal guinea pig myocardium has a current density and ATP sensitivity similar to those of the adult, but is not activated even under hypoxic conditions because the energy metabolism of the fetal myocardium is less dependent on oxidative phosphorylation. This makes the action potential and contractile force of fetal myocardia resistant to hypoxia.

Acknowledgments This study was supported in part by Ministry of Education, Culture, Sports, Science and Technology (MEXT) KAKENHI Grant numbers JP15K08247, JP17K08607 and JP17K15460.

Conflict of Interest The authors declare no conflict of interest.

\section{REFERENCES}

1) Tohse N, Seki S, Kobayashi T, Tsutsuura M, Nagashima M, Yamada Y. Development of excitation-contraction coupling in cardiomyocytes. Jpn. J. Physiol., 54, 1-6 (2004).

2) Tanaka H, Namekata I, Nouchi H, Shigenobu K, Kawanishi T, Takahara A. New aspects for the treatment of cardiac diseases based on the diversity of functional controls on cardiac muscles: diversity in the excitation-contraction mechanisms of the heart. $J$. Pharmacol. Sci., 109, 327-333 (2009).

3) Louch WE, Koivumäki JT, Tavi P. Calcium signaling in developing cardiomyocytes: implications for model systems and disease. $J$. Physiol., 593, 1047-1063 (2015).

4) Agata N, Tanaka H, Shigenobu K. Inotropic effects of ryanodine and nicardipine on fetal, neonatal and adult guinea-pig myocardium. Eur. J. Pharmacol., 260, 47-55 (1994).

5) Agata N, Tanaka H, Shigenobu K. Developmental changes in action potential properties of the guinea-pig myocardium. Acta Physiol. Scand., 149, 331-337 (1993).

6) Kato Y, Masumiya H, Agata N, Tanaka H, Shigenobu K. Developmental changes in action potential and membrane currents in fetal, neonatal and adult guinea-pig ventricular myocytes. J. Mol. Cell.
Cardiol., 28, 1515-1522 (1996).

7) Itoh H, Naito Y, Tomita M. Simulation of developmental changes in action potentials with ventricular cell models. Syst. Synth. Biol., 1, 11-23 (2007).

8) Noma A, Takano M. The ATP-sensitive $\mathrm{K}^{+}$channel. Jpn. J. Physiol., 41, 177-187 (1991).

9) Kefaloyianni E, Bao L, Rindler MJ, Hong M, Patel T, Taskin E, Coetzee WA. Measuring and evaluating the role of ATP-sensitive $\mathrm{K}^{+}$channels in cardiac muscle. J. Mol. Cell. Cardiol., 52, 596-607 (2012).

10) Matsuda T, Okazaki K, Kato Y, Tanaka H, Shigenobu KK. ${ }^{+}$channel-opening properties of a novel compound, NIP-121, in guinea pig myocardium as compared with those of cromakalim. J. Cardiovasc. Pharmacol., 26, 608-613 (1995).

11) Tanaka H, Okazaki K, Shigenobu K. Cardioprotective effects of NIP-121, a novel ATP-sensitive potassium channel opener, during ischemia and reperfusion in coronary perfused guinea pig myocardium. J. Cardiovasc. Pharmacol., 27, 695-701 (1996).

12) Mcdonald TF, MacLeod DP. Anoxia-recovery cycle in ventricular muscle: action potential duration, contractility and ATP content. Pflugers Arch., 325, 305-322 (1971).

13) Agata N, Kato Y, Tanaka H, Shigenobu K. Differential effects of hypoxia on electrical and mechanical activities of isolated ventricular muscles from fetal and adult guinea-pigs. Gen. Pharmacol., 25, 15-18 (1994).

14) Namekata I, Shimada H, Kawanishi T, Tanaka H, Shigenobu K. Reduction by SEA0400 of myocardial ischemia-induced cytoplasmic and mitochondrial $\mathrm{Ca}^{2+}$ overload. Eur. J. Pharmacol., 543, 108-115 (2006).

15) Hamaguchi S, Kawakami Y, Honda Y, Nemoto K, Sano A, Namekata I, Tanaka H. Developmental changes in excitation-contraction mechanisms of the mouse ventricular myocardium as revealed by functional and confocal imaging analyses. J. Pharmacol. Sci., 123, 167-175 (2013).

16) Lopaschuk GD, Jaswal JS. Energy metabolic phenotype of the cardiomyocyte during development, differentiation, and postnatal maturation. J. Cardiovasc. Pharmacol., 56, 130-140 (2010).

17) Barrie SE, Harris P. Myocardial enzyme activities in guinea pigs during development. Am. J. Physiol., 233, H707-H710 (1977).

18) Rolph TP, Jones CT. Regulation of glycolytic flux in the heart of the fetal guinea pig. J. Dev. Physiol., 5, 31-49 (1983).

19) Hirakow R, Gotoh T. Quantitative studies on the ultrastructural differentiation and growth of mammalian cardiac muscle cells. Acta Anatomica, 108, 230-237 (1980).

20) Sano HI, Toki T, Naito Y, Tomita M. Developmental changes in the balance of glycolytic ATP production and oxidative phosphorylation in ventricular cells: a simulation study. J. Theor. Biol., 419, 269-277 (2017).

21) Sekine K, Kagawa Y, Maeyama E, Ota H, Haraguchi Y, Matsuura $\mathrm{K}$, Shimizu T. Oxygen consumption of human heart cells in monolayer culture. Biochem. Biophys. Res. Commun., 452, 834-839 (2014)

22) Tohyama S, Hattori F, Sano M, Hishiki T, Nagahata $Y$, Matsuura $T$, Hashimoto H, Suzuki T, Yamashita H, Satoh Y, Egashira T, Seki T, Muraoka N, Yamakawa H, Ohgino Y, Tanaka T, Yoichi M, Yuasa S, Murata M, Suematsu M, Fukuda K. Distinct metabolic flow enables large-scale purification of mouse and human pluripotent stem cellderived cardiomyocytes. Cell Stem Cell, 12, 127-137 (2013).

23) Weiss J, Hiltbrand B. Functional compartmentation of glycolytic versus oxidative metabolism in isolated rabbit heart. J. Clin. Invest., 75, 436-447 (1985)

24) Weiss JN, Lamp ST. Glycolysis preferentially inhibits ATP-sensitive $\mathrm{K}^{+}$channels in isolated guinea pig cardiac myocytes. Science, $\mathbf{2 3 8}$, 67-69 (1987).

25) Weiss JN, Lamp ST. Cardiac ATP-sensitive $\mathrm{K}^{+}$channels. Evidence for preferential regulation by glycolysis. J. Gen. Physiol., 94, 911935 (1989). 